\title{
Comprehensive Analyses of miRNA-mRNA Network and Potential Drugs in Idiopathic Pulmonary Arterial Hypertension
}

\author{
Chan Li $\mathbb{D}^{1}$ Zeyu Zhang $\mathbb{D}^{2}{ }^{2}$ Qian Xu $\mathbb{D}^{3},{ }^{3}$ and Ruizheng Shi $\mathbb{D}^{1}$ \\ ${ }^{1}$ Department of Cardiovascular Medicine, Xiangya Hospital, Central South University, Changsha, Hunan, China \\ ${ }^{2}$ Department of Hepatobiliary Surgery, Xiangya Hospital, Central South University, Changsha, Hunan, China \\ ${ }^{3}$ Department of Cardiovascular Surgery, Xiangya Hospital, Central South University, Changsha, Hunan, China
}

Correspondence should be addressed to Ruizheng Shi; xyshiruizheng@csu.edu.cn

Received 27 March 2020; Revised 26 May 2020; Accepted 16 June 2020; Published 4 July 2020

Academic Editor: Bilal Alatas

Copyright ( 92020 Chan Li et al. This is an open access article distributed under the Creative Commons Attribution License, which permits unrestricted use, distribution, and reproduction in any medium, provided the original work is properly cited.

\begin{abstract}
Introduction. Idiopathic pulmonary arterial hypertension (IPAH) is a severe cardiopulmonary disease with a relatively low survival rate. Moreover, the pathogenesis of IPAH has not been fully recognized. Thus, comprehensive analyses of miRNA-mRNA network and potential drugs in IPAH are urgent requirements. Methods. Microarray datasets of mRNA and microRNA (miRNA) in IPAH were searched and downloaded from Gene Expression Omnibus (GEO). Differentially expressed genes (DEGs) and differentially expressed miRNAs (DEMIs) were identified. Then, the DEMI-DEG network was conducted with associated comprehensive analyses including Gene Ontology (GO) analysis, Kyoto Encyclopedia of Genes and Genomes (KEGG) pathway enrichment analysis, and protein-protein interaction (PPI) network analysis, while potential drugs targeting hub genes were investigated using L1000 platform. Results. 30 DEGs and 6 DEMIs were identified in the lung tissue of IPAH. GO and KEGG pathway analyses revealed that these DEGs were mostly enriched in antimicrobial humoral response and African trypanosomiasis, respectively. The DEMI-DEG network was conducted subsequently with 4 DEMIs (hsa-miR-34b-5p, hsa-miR-26b-5p, hsa-miR205-5p, and hsa-miR-199a-3p) and 16 DEGs, among which 5 DEGs (AQP9, SPP1, END1, VCAM1, and SAA1) were included in the top 10 hub genes of the PPI network. Nimodipine was identified with the highest CMap connectivity score in L1000 platform. Conclusion. Our study conducted a miRNA-mRNA network and identified 4 miRNAs as well as 5 mRNAs which may play important roles in the pathogenesis of IPAH. Moreover, we provided a new insight for future therapies by predicting potential drugs targeting hub genes.
\end{abstract}

\section{Introduction}

Idiopathic pulmonary arterial hypertension (IPAH), a rare but life-threatening cardiopulmonary disease without any known associated disease or genetic cause, is characterized by progressively increased pulmonary artery pressure (PAP) and pulmonary vascular resistance (PVR) [1, 2]. New therapies such as calcium channel antagonists, endothelin receptor antagonists, and phosphodiesterase type 5 (PDE5) inhibitors have been applied to IPAH patients in the last two decades and have improved the survival rate of patients who are specifically sensitive to the drugs $[3,4]$. For patients who failed to respond adequately to medical therapies, lung transplantation remained the last option. For now, IPAH is still supposed to be an incurable disease resulting in progressive loss of quality of life with a total 5year survival rate of $51 \%$ and a 5 -year survival rate of $47 \%$ despite receiving lung transplantation $[5,6]$. Thus, comprehensive analyses of potential mechanisms and searching for more possible therapies in IPAH are urgently needed.

microRNA (miRNA) is a type of small, noncoding RNA, which negatively regulates the expression of targeted genes via posttranscriptional regulation [7]. Previous studies have uncovered that dysregulation of miRNAs such as miR-204 and miR-21 was associated with the pathobiology of IPAH [8-10], suggesting miRNAs maybe novel therapeutic targets. However, few studies explore the gene targets and molecularregulated network of IPAH-relevant miRNAs, which are necessary for the development of miRNA-based treatments [11]. In this regard, systematic analyses combining transcriptomic 
TABLE 1: Details of datasets related to IPAH patients.

\begin{tabular}{|c|c|c|c|c|c|c|c|}
\hline & GEO ID & Platform & Organism & Experiment type & Samples (case vs. control) & Country & Year \\
\hline mRNA & GSE117261 & GPL6244 & Homo sapiens & Expression profiling by array & 32 vs. 25 & $\begin{array}{c}\text { United States } \\
\text { of America }\end{array}$ & 2018 \\
\hline & GSE113439 & GPL6244 & Homo sapiens & Expression profiling by array & 6 vs. 11 & Canada & 2018 \\
\hline miRNA & GSE67597 & GPL18402 & Homo sapiens & Noncoding RNA profiling by array & 7 vs. 8 & $\begin{array}{l}\text { United States } \\
\text { of America }\end{array}$ & 2015 \\
\hline
\end{tabular}

IPAH: idiopathic pulmonary arterial hypertension; GEO; Gene Expression Omnibus.

data with miRNA data are demanded to identify essential pathways and potential drugs in IPAH.

Bioinformatic analysis has been widely used to investigate potential mechanisms in the pathology of disease since it was developed [12-14]. Recently, a few diagnostic biomarkers for IPAH have been found by application of bioinformatic analysis [15], but the miRNA expression in IPAH and their functional roles as well as mRNA targets are poorly studied.

In this study, microarray datasets of IPAH were searched and downloaded from Gene Expression Omnibus (GEO). miRNA-mRNA network was established accompanied with associated comprehensive analyses in order to better understand the mechanisms of IPAH. Additionally, potential drugs targeting hub genes were investigated to contribute to future therapies of IPAH.

\section{Methods}

2.1. Data Resources. The mRNA and miRNA expression profiles of IPAH patients from Gene Expression Omnibus (https://www.ncbi.nlm.nih.gov/geoprofiles/) and ArrayExpress (https://www.ebi.ac.uk/arrayexpress/) were searched. GSE113439 and GSE117261 with mRNA expression profile and GSE67597 with miRNA expression profile were identified in GEO, while no related profile was found in ArrayExpress. Subsequently, secondary PAH patients with other diseases were excluded. Finally, GSE113439 with 6 IPAH and 11 controls, GSE117261 with 32 IPAH and 25 controls, and GSE67597 with 7 IPAH and 8 controls were included in this study. Additional approval by an ethics committee was not necessary because the datasets included in the current study were downloaded from public databases.

2.2. Identification of DEGs and DEMIs. R-platform (http://Rproject.org) and limma package [16] were used to screen the differentially expressed genes (DEGs) and differentially expressed miRNAs (DEMIs) between IPAH and healthy controls. $\left|\log _{2} \mathrm{FC}\right|>1$ was considered the threshold for different expressions, and statistical difference was defined as adjusted $P$ value $<0.05$. Those with $\log _{2} \mathrm{FC}<0$ were considered as downregulated genes, while those with $\log _{2} \mathrm{FC}>0$ were considered as upregulated genes. The visualization of DEGs and DEMIs was realized in the volcano plot and heat map by pheatmap package in $\mathrm{R}$ platform. Two series (GSE113439 and GSE117261) containing mRNA expression profile were from the same platform ([HuGene-1_0-st] Affymetrix Human Gene 1.0 ST Array [transcript (gene) version]) and merged into one for analyses after batch normalization realized by sva package in $\mathrm{R}$ platform.

2.3. Functional Enrichment Analyses. R package clusterProfiler [17] was applied to conduct Gene Ontology (GO) analysis and Kyoto Encyclopedia of Genes and Genomes (KEGG) pathway enrichment analysis to identify the potential biological function of DEGs and analyze the enriched pathway of the key DEGs. Adjusted $P$ value $<0.05$ was considered statistically significant.

2.4. PPI Network Analysis. The online tool, STRING (http:// string-db.org/) [18], was employed to establish proteinprotein interaction (PPI) network of DEG-encoded proteins with a confidence score $>0.40$. Subsequently, hub genes and core modules were selected and visualized by Cytoscape software (V3.5.1; http://cytoscape.org/) [19].

2.5. Prediction of miRNA-Targeted Gene and DEMI-DEG Network. To better understand the function of DEMI, miRDB (http://www.mirdb.org/), TargetScan (http://www .targetscan.org/vert_72/), TargetMiner (https://www.isical .ac.in/ bioinfo_miu), and miRWalk (http://zmf.umm.uniheidelberg.de/apps/zmf/mirwalk) were used to predict target genes. Predicted genes in these four databases were pooled into a solitary database, and the intersection of DEGs and the database were regarded as significantly differentially expressed target genes. Furthermore, the DEMI-DEG network was constructed by Cytoscape software.

2.6. Prediction of Potential Drugs for IPAH. L1000 platform (https://clue.io/) was used to explore potential drugs toward IPAH for pharmaceutical development [20]. Upregulated DEGs involved in the DEMI-DEG network were submitted to the L1000 platform for prediction of potential drugs for IPAH. Drugs with the CMap connectivity score of +90 or higher, and of -90 or lower, were considered to be potential effective drugs.

\section{Results}

3.1. Identification of DEGs and DEMIs. The basic information of the datasets related to IPAH is shown in Table 1. The expression of different genes was calculated according to mapped probes, and average value was applied if multiple probes matched the same gene. Overall, 20146 genes were analyzed in GSE113439 and GSE117261. 30 DEGs were found between IPAH patients and the control group, among which 14 were upregulated and 16 were downregulated (Figure 1(a)). Furthermore, 2006 miRNAs were analyzed 


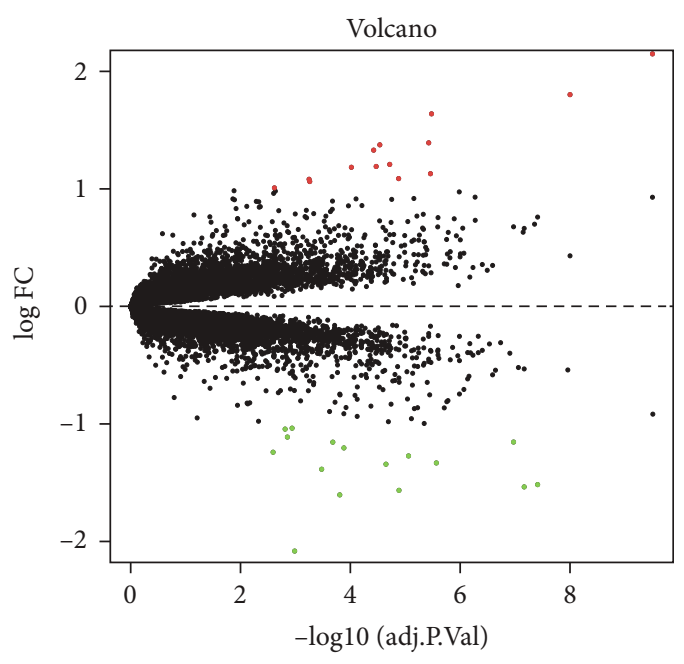

(a)

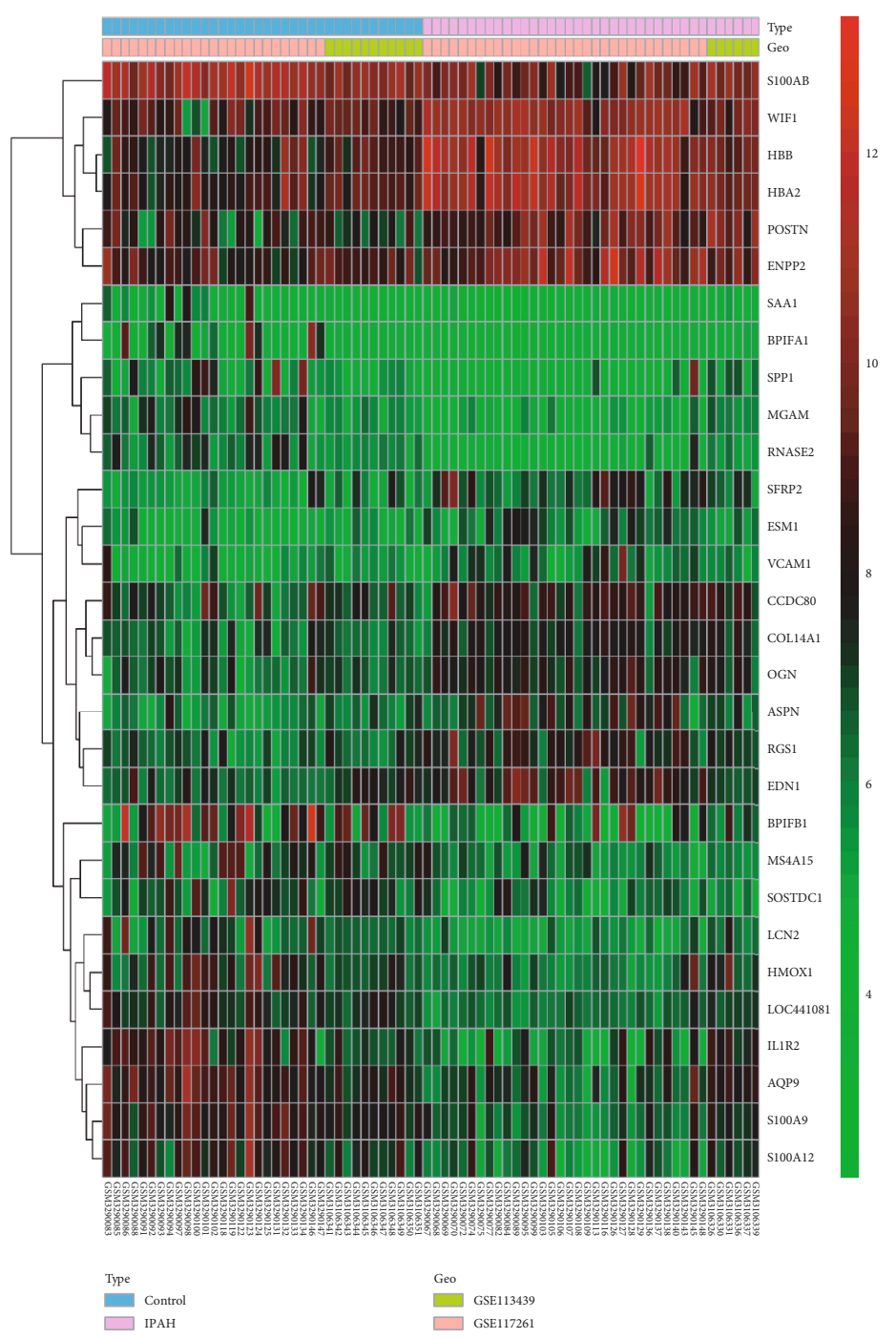

(b)

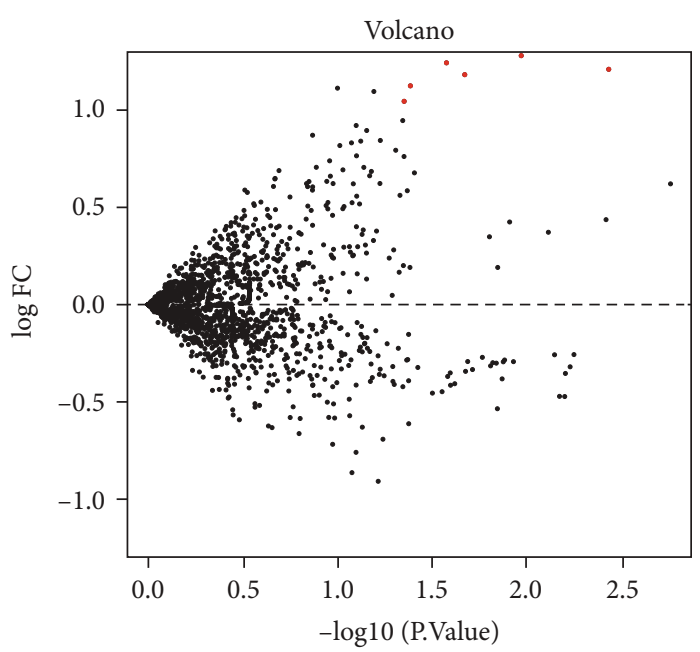

(c)

Figure 1: (a) Volcano plot of DEGs in IPAH. Green represents downregulated DEGs; red represents upregulated DEGs; and black represents no difference. (b) Heat map of the DEGs in IPAH compared with normal controls. Red represents greater expression and green represents less expression. (c) Volcano plot of DEMIs in IPAH. DEG: differentially expressed gene; IPAH: idiopathic pulmonary arterial hypertension; DEMI: differentially expressed miRNA. 


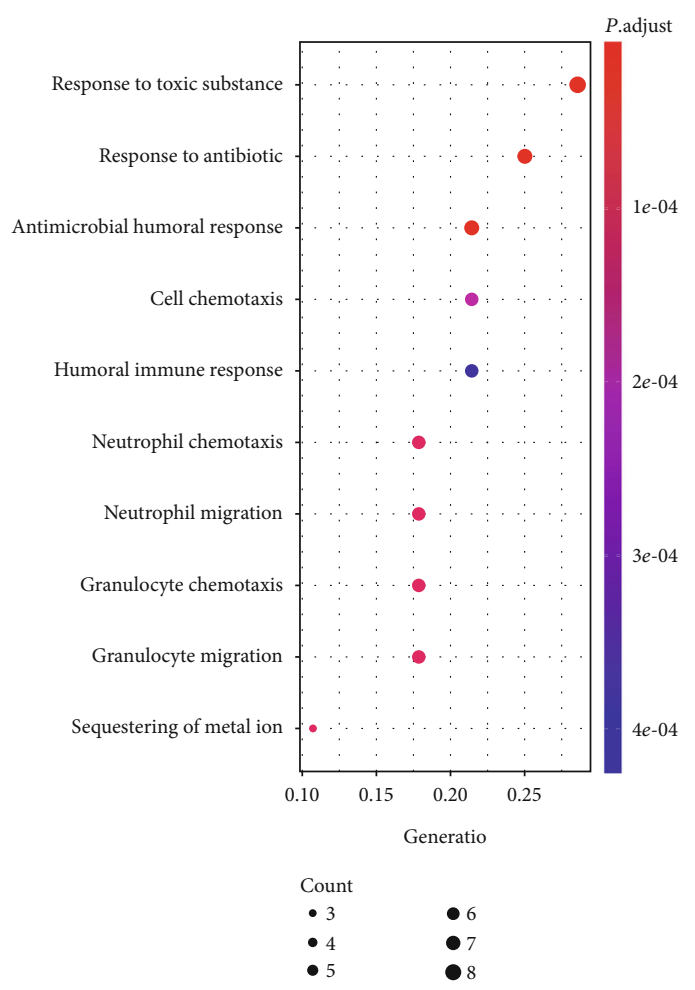

(a)

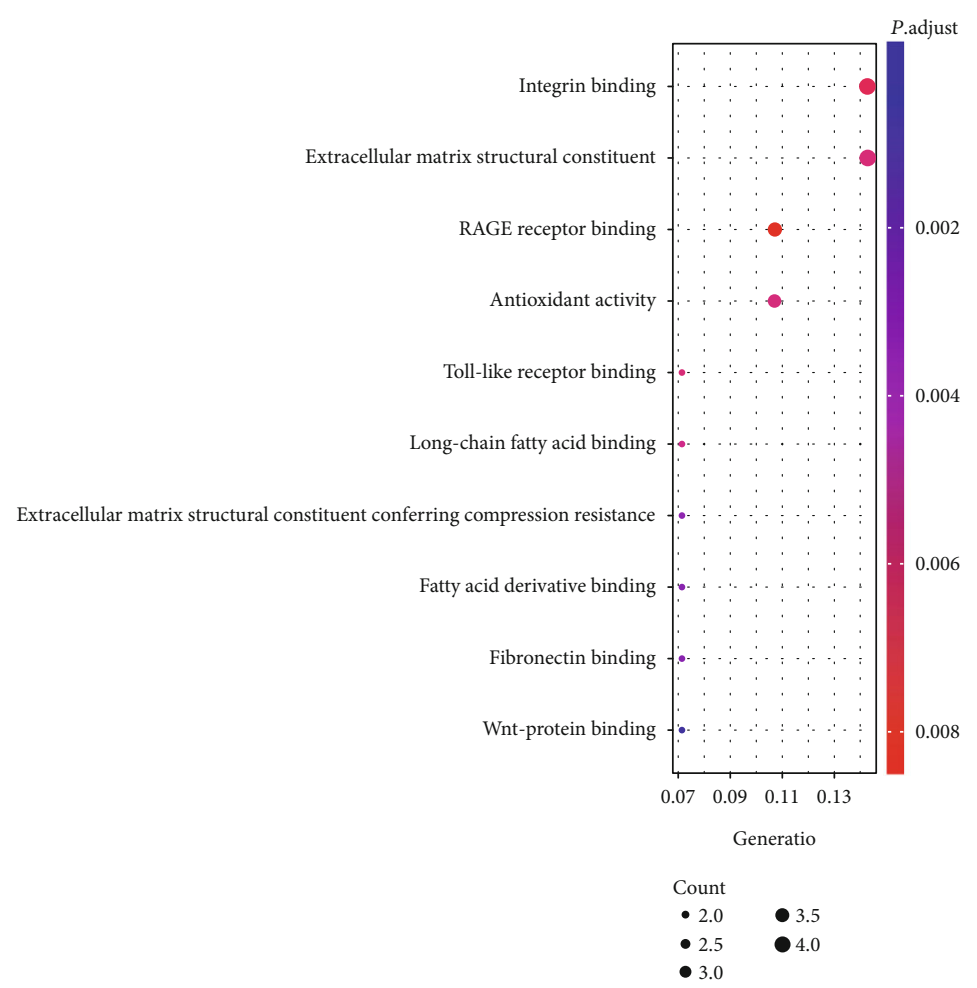

(c)

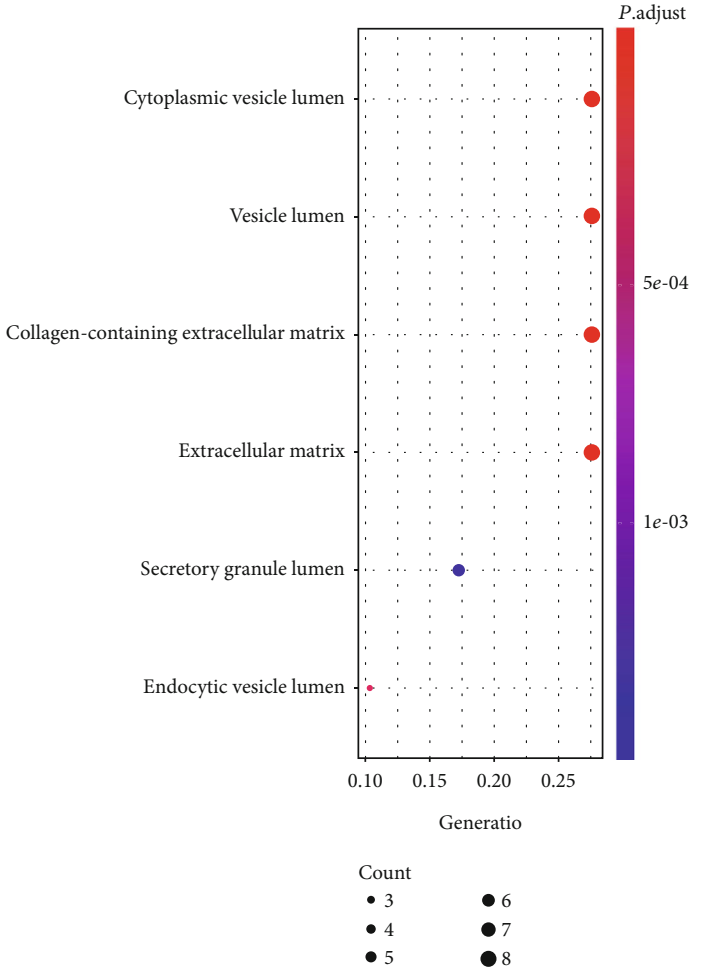

(b)

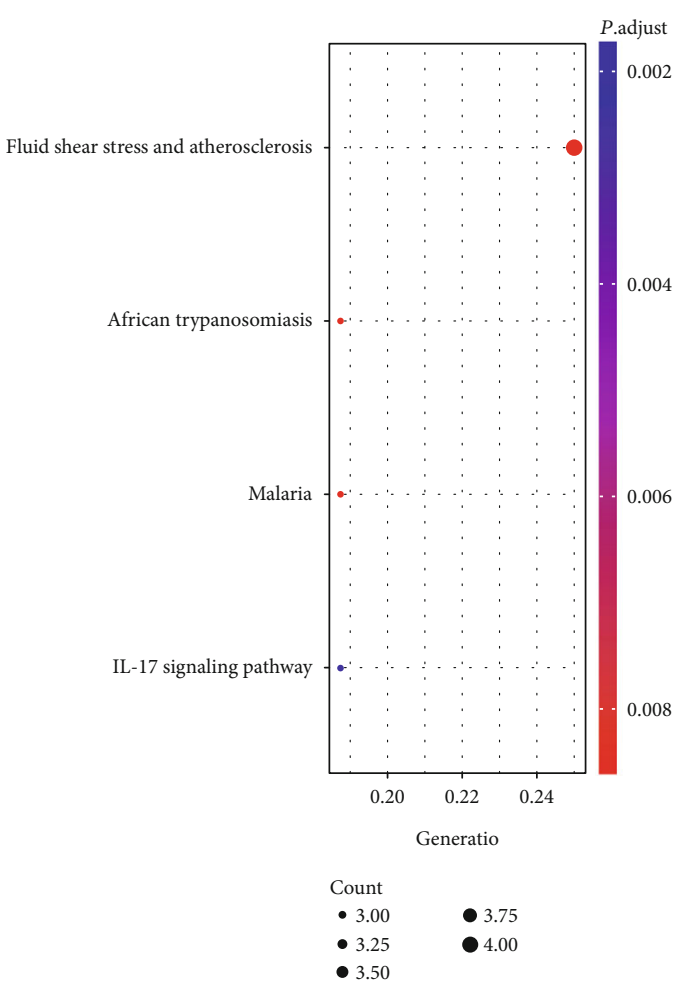

(d)

Figure 2: The top 10 enriched Gene Ontology terms in biological process (a), cellular component (b), molecular function (c) and enriched Kyoto Encyclopedia of Genes and Genomes pathway (d) of DEGs. DEG: differentially expressed gene. 


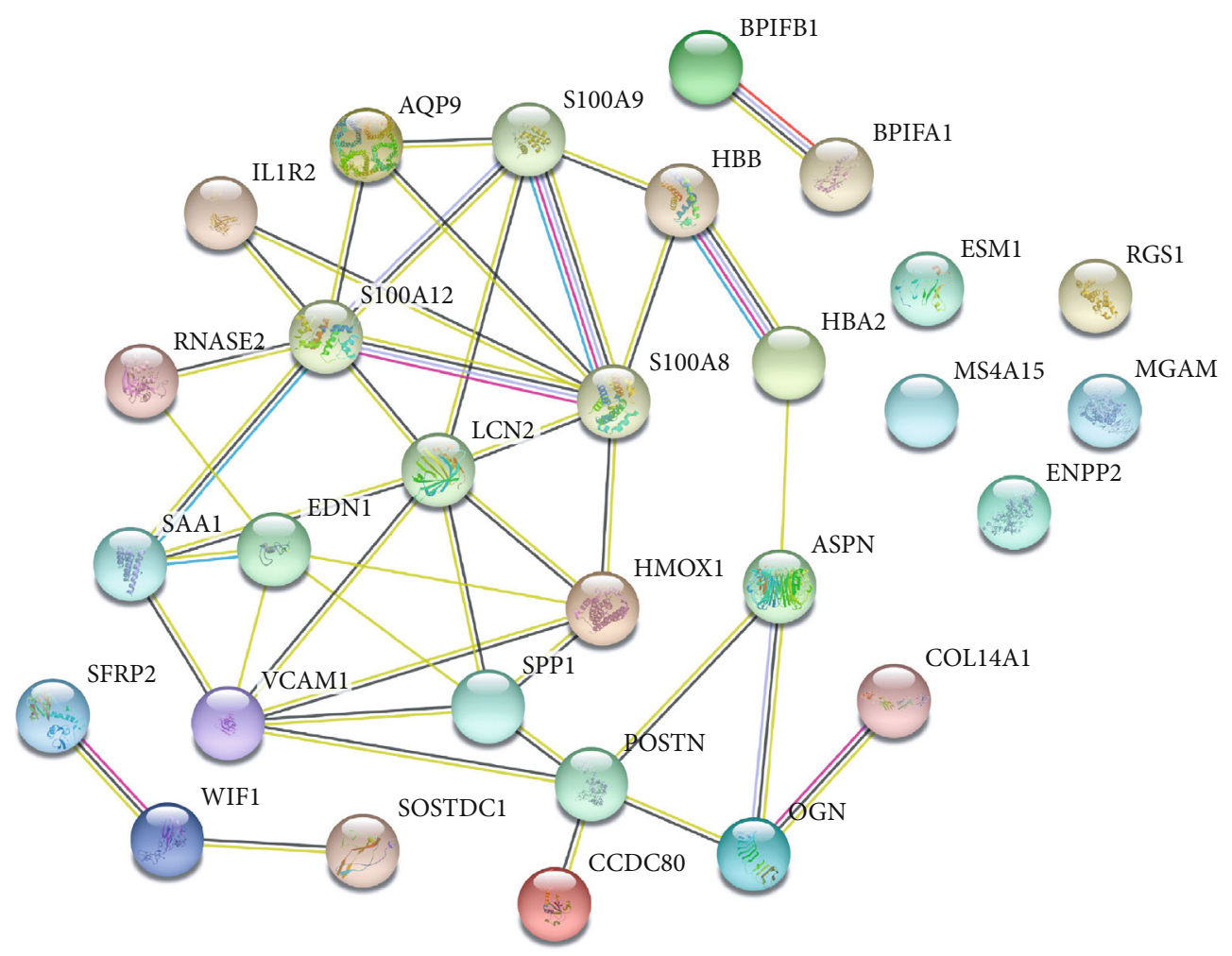

(a)

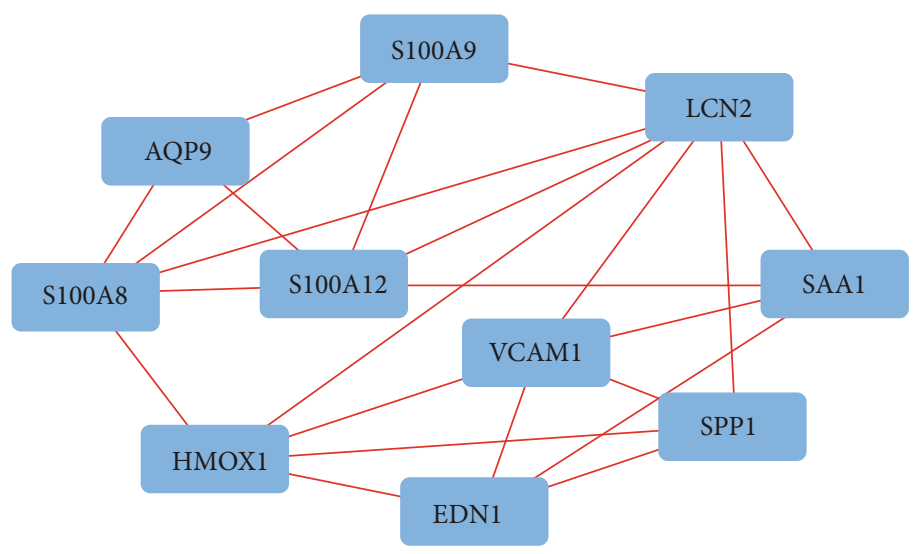

(b)

FIgURE 3: Protein-protein interaction network analysis (a) and hub genes (b) identified by Cytoscape.

and 6 DEMIs were found, indicating these 6 DEMIs may participate in the pathogenesis of IPAH (Figure 1(b)). Details of DEGs and DEMIs between IPAH patients and the control are showed in Supplement Tables 1 and 2, respectively.

3.2. Functional Enrichment Analyses. Subsequently, functional enrichment analyses were performed to reveal the function of DEGs and the results are shown in Figure 2.

Go enrichment analysis revealed that DEG-related biological processes (BP) were mostly enriched in antimicrobial humoral response $(P$ value $<0.0001)$, neutrophil chemotaxis $(P$ value $<0.0001)$, and neutrophil migration $(P$ value $<$ 0.0001), while cellular components (CC) were mainly enriched in cytoplasmic vesicle lumen $(P$ value $<0.0001)$, vesicle lumen $(P$ value $<0.0001)$, and collagen-containing extracellular matrix $(P$ value $<0.0001)$. In addition, molecular function (MF) analyses suggested that DEGs were involved in RAGE receptor binding $(P$ value $=0.0016)$, integrin binding $(P$ value $=0.0159)$ and Toll-like receptor binding $(P$ value $=0.0029)$.

To investigate the crucial pathways of these DEGs, KEGG pathways analysis was performed and the significant pathways are shown in Figure 2. The DEGs were enriched in the pathway of African trypanosomiasis $(P$ value $=0.0020)$, malaria $(P$ value $=0.0020)$, fluid shear stress and atherosclerosis $(P$ value $=$ $0.0020)$, and IL-17 signaling pathway $(P$ value $=0.0085)$.

3.3. PPI Network Analysis. Using the STRING database, we performed PPI network analysis for DEGs. As we have shown in Figure 3, 24 nodes were mainly identified. AQP9, 


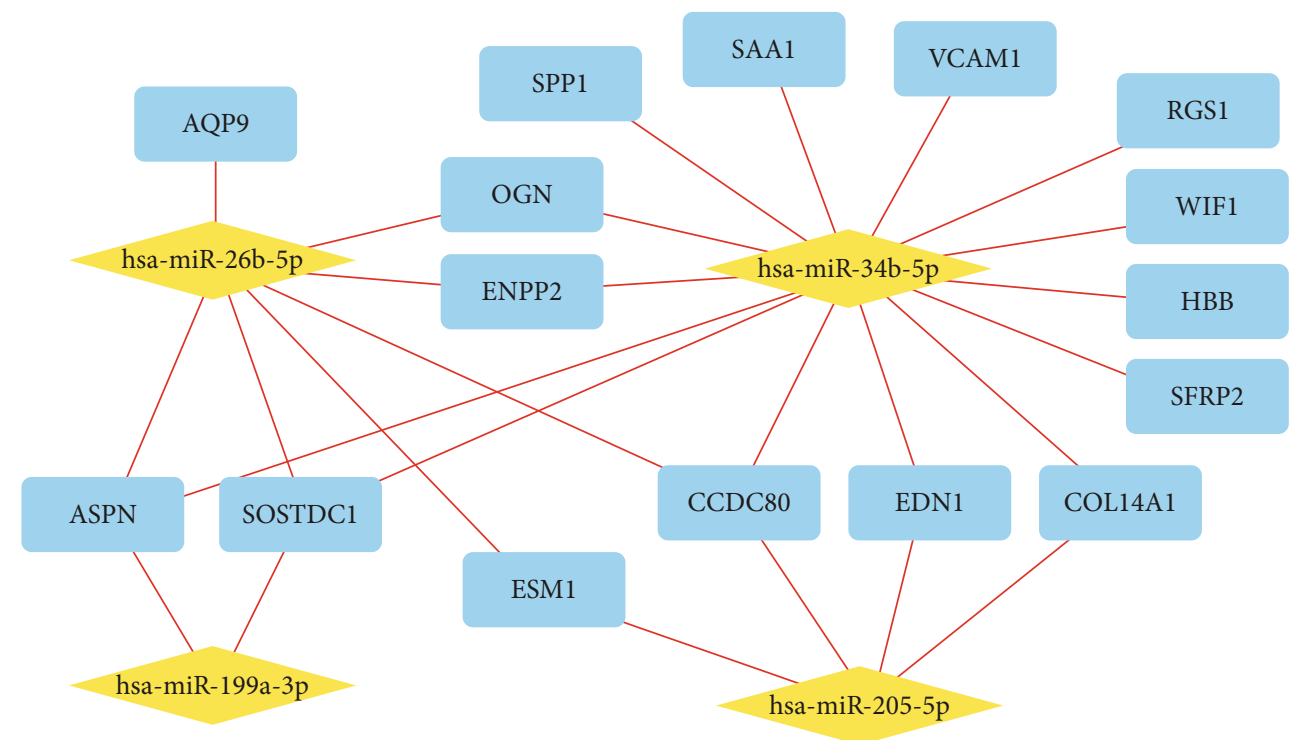

FIGURE 4: DEMI-DEG network. Rhombus represents miRNA while rectangle represents mRNA. DEG: differentially expressed gene; DEMI: differentially expressed miRNA.

TABLE 2: Details of the 16 DEGs included in DEMI-DEG network.

\begin{tabular}{lccc}
\hline Gene & Ensembl ID & $\log _{2}$ FC & Adjusted $P$ value \\
\hline HBB & ENSG00000244734 & 2.1829 & $3.26 E-10$ \\
COL14A1 & ENSG00000187955 & 1.1296 & $3.55 E-06$ \\
WIF1 & ENSG00000156076 & 1.3922 & $3.82 E-06$ \\
OGN & ENSG00000106809 & 1.0880 & $1.34 E-05$ \\
RGS1 & ENSG00000090104 & 1.2087 & $1.95 E-05$ \\
AQP9 & ENSG00000103569 & -1.3423 & $2.29 E-05$ \\
ASPN & ENSG00000106819 & 1.3752 & $2.96 E-05$ \\
ESM1 & ENSG00000164283 & 1.1911 & $3.45 E-05$ \\
SFRP2 & ENSG00000145423 & 1.3301 & $3.83 E-05$ \\
ENPP2 & ENSG00000136960 & 1.1842 & $9.72 E-05$ \\
SAA1 & ENSG00000173432 & -1.1547 & 0.0002 \\
EDN1 & ENSG00000078401 & 1.0631 & 0.0006 \\
VCAM1 & ENSG00000162692 & 1.0820 & 0.0006 \\
SOSTDC1 & ENSG00000171243 & -1.1114 & 0.0014 \\
CCDC80 & ENSG00000091986 & 1.0089 & 0.0024 \\
SPP1 & ENSG00000118785 & -1.2404 & 0.0026
\end{tabular}

DEG: differentially expressed gene; DEMI: differentially expressed miRNA; FC: fold change.

EDN1, S100A12, S100A8, S100A9, SPP1, HMOX1, VCAM1, LCN2, and SAA1 were the top 10 hub genes.

3.4. Prediction of miRNA-Targeted Gene and DEMI-DEG Network. In order to further identify important miRNAmRNA-regulated axes in IPAH, four databases (miRDB, TargetScan, TargetMiner, and miRWalk) were employed to predict the target genes of DEMIs. 9095 genes were predicted as target genes of 6 DEMIs. GO and KEGG analyses of those genes were performed with results shown in Supplement Figure 1. Among the target genes of DEMIs, 16 were intersected with DEGs (HBB, COL14A1, WIF1, OGN, RGS1, ASPN, ESM1, SFRP2, ENPP2, EDN1, VCAM1,
TABle 3: Details of the 4 DEMIs included in the DEMI-DEG network.

\begin{tabular}{lccc}
\hline Gene & miRBase & $\log _{2} \mathrm{FC}$ & $P$ value \\
\hline hsa-miR-205-5p & MIMAT0000266 & 1.2088 & 0.0038 \\
hsa-miR-199a-3p & MIMAT0000232 & 1.2975 & 0.0109 \\
hsa-miR-34b-5p & MIMAT0000685 & 1.2421 & 0.0270 \\
hsa-miR-26b-5p & MIMAT0000083 & 1.1240 & 0.0418 \\
\hline
\end{tabular}

DEG: differentially expressed gene; DEMI: differentially expressed miRNA; FC: fold change.

CCDC80, AQP9, SAA1, SOSTDC1, and SPP1), which were used to construct the DEMI-DEG network (Figure 4). The network consisted of 4 DEMIs, 16 predicted target DEGs, and 27 edges, while hsa-miR-99a-5p and hsa-miR-30a-5p were connected with no predicted target DEGs and were excluded from the network. hsa-miR-34b-5p was related to most of the predicted target DEGs (14 edges), followed by hsa-miR-26b-5p (7 edges), hsa-miR-205-5p (4 edges), and hsa-miR-199a-3p (2 edges). In the other hand, ASPN, CCDC80, and SOSTDC1 were connected with 3 DEMIs, respectively. Details of DEGs and DEMIs in the DEMIDEG network are showed in Tables 2 and 3, respectively.

3.5. Prediction of Potential Drugs for IPAH. The 12 upregulated DEGs in the DEMI-DEG network were uploaded to the L1000 platform to search for potential drugs, and the top 20 ranked by CMap connectivity score are listed in Table 4.

\section{Discussion}

Though new therapies have been developed in recent years, IPAH remains a severe disease with continuous suffering to patients and society. Therefore, better understanding of potential mechanisms in the pathogenesis of IPAH may shed a light for future studies. 
TABLE 4: The top 10 and bottom 10 chemical compounds identified by L1000 platform.

\begin{tabular}{|c|c|c|}
\hline Name & CMap connectivity score & Description \\
\hline Nimodipine & 99.89 & Calcium channel blocker \\
\hline GW-501516 & 99.82 & PPAR receptor agonist \\
\hline Icilin & 99.82 & TRPV agonist \\
\hline Fexofenadine & 99.82 & Histamine receptor antagonist \\
\hline Modafinil & 99.79 & Adrenergic receptor agonist \\
\hline Guanabenz & 99.79 & Alpha-2 selective adrenergic agonist \\
\hline Mianserin & 99.75 & Serotonin receptor antagonist \\
\hline CGP-53353 & 99.75 & EGFR inhibitor \\
\hline TUL-XXI039 & 99.72 & Serine/threonine kinase inhibitor \\
\hline Diphenoxylate & 99.68 & Opioid receptor agonist \\
\hline Huperzine-a & -99.46 & Acetylcholinesterase inhibitor \\
\hline Perindopril & -99.47 & ACE inhibitor \\
\hline Solanine & -99.47 & Acetylcholinesterase inhibitor \\
\hline Phenothiazine & -99.51 & Dopamine receptor antagonist \\
\hline Tyrphostin-AG-1295 & -99.68 & PDGFR receptor inhibitor \\
\hline Y-27152 & -99.72 & Potassium channel activator \\
\hline Lenalidomide & -99.79 & Antineoplastic \\
\hline Androstenedione & -99.89 & Cytochrome P450 inhibitor \\
\hline Repaglinide & -99.89 & Insulin secretagogue \\
\hline Neurodazine & -99.89 & Neurogenesis of nonpluripotent $\mathrm{C} 2 \mathrm{C} 12$ myoblast inducer \\
\hline
\end{tabular}

In the present study, 30 DEGs, mostly related to immune and inflammatory response such as neutrophil chemotaxis and migration, integrin binding and Toll-like receptor binding, and significantly enriched in inflammation pathways like IL-17 signaling pathway, and 6 DEMIs were found in IPAH. The DEMI-DEG network was conducted subsequently with 4 DEMIs (hsa-miR-34b-5p, hsa-miR-26b-5p, hsa-miR-205$5 \mathrm{p}$, and hsa-miR-199a-3p) and 16 DEGs. We found that 5 DEGs (AQP9, SPP1, END1, VCAM1, and SAA1) were included in the top 10 hub genes of the PPI network, indicating those factors may play important roles in the pathogenesis of IPAH. Finally, drugs including nimodipine were identified. To our knowledge, this is the first study that conducted the network of mRNA and miRNA and predicted potential drugs in IPAH, which provides a foundation for further research and development of therapy.

Previous studies have revealed the important role of the miRNAs and mRNAs that is identified in our DEMI-DEG network in the pathogenesis of IPAH. Wu et al. identified dysregulated miRNAs in IPAH via miRNA profiling and qRT-PCR. miR-26b-5p and miR-199a-3p were detected as two of the top 5 most significantly increased miRNAs targeting major PAH related pathways including $\mathrm{Wnt} / \beta$-catenin in end-stage IPAH [21]. In pulmonary vascular smooth muscle cells (PASMCs), miR-205-5p was reported to suppress cell proliferation by targeting MICAL2-mediated Erk1/2 signaling, which may be regarded as a therapeutic target in $\mathrm{PAH}$ [22]. For mRNA, 5 mRNAs of the top 10 hub genes in the PPI network were included in our DEMI-DEG network. Among them, endothelin-1 (EDN1) is a potent vasoconstrictor and its receptors are therapeutic targets in the treatment of $\mathrm{PAH}[23,24]$. It was able to promote contraction via increasing intracellular $\mathrm{Ca}^{2+}$ and increasing PASMC proliferation and migration, leading to vascular remodeling, which is a key mechanism underlying PAH [25-27]. Vascular cell adhesion molecule 1 (VCAM1), an adhesion molecule mediating leukocyte transmigration and increasing tissue inflammation [28], was found to increase in both patients and animal models of IPAH $[29,30]$. Aquaporin (AQP), a family of water-selective membrane channels promoting endothelial cell migration and angiogenesis [31], was found to be overexpressed in PAH patients $[32,33]$, indicating its potential role in the treatment of PAH. Secreted phosphoprotein 1 (SPP1, also known as osteopontin or OPN), a key mediator secreted by SMCs, contributes to the genesis and progression of pulmonary hypertension by enhancing PVSMC proliferation [34-36]. It is upregulated in the lung tissues of patients, and the SPP1 expression level is associated with the severity of PAH [37-39]. These results demonstrated that SPP1 may be a prognostic marker as well as a therapeutic target in $\mathrm{PAH}$.

However, some of the factors in our DEMI-DEG network were poorly studied. The role of hsa-miR-34b-5p, which was connected with most of the predicted target DEGs, has not been studied in IPAH before. miR-34b was known to affect cell proliferation and adhesion-independent growth in several types of cancer [40, 41]. Lin et al. previously discovered DNA methylation of miR-34b-regulated vascular calcification by targeting Notch1 [42]. Furthermore, serum amyloid A1 (SAA1), an acute phase protein, is primarily synthesized in the liver and highly expressed when inflammatory response occurs [43]. The elevated SAA1 concentration was associated with cardiovascular risk [44], carotid intima 
media thickness [45], and smooth muscle cell homeostasis [46] according to previous researches. Whether and how these factors contribute to IPAH need to be investigated in future studies.

The calcium channel blocker is an effective treatment for IPAH patients with a positive vasodilator response, which dramatically improves the survival of those patients and has been widely applied recent years $[47,48]$. Nimodipine, a kind of calcium channel blocker, was identified as the top of the potential drugs in our research. However, other calcium channel blockers were not predicted with a high rank, so whether nimodipine has a clinical benefit cannot be determined. Furthermore, other drugs in the prediction list, which were hardly researched in IPAH before, may serve as potential directions for future development of therapies in IPAH.

There are some limitations in our study. Firstly, only two mRNA expression profiles and one miRNA expression profile were found in GEO; the relatively small number of samples may make the results less convincing. Secondly, since the expression profiles of miRNA and mRNA were obtained from different datasets in this study, coexpression analysis cannot be performed currently, which prevents us from obtaining a more accurate miRNA-mRNA regulation network. In addition, PCR or WB should be done to further confirm our results, and the relationship between miRNAs and mRNAs in our DEMI-DEG network should be validated via in vivo or in vitro experiments.

\section{Conclusion}

Our study initially conducted a miRNA-mRNA network including 4 miRNAs and 5 mRNAs to systematically analyze the pathogenesis of IPAH and provided a new insight for future therapies by predicting potential drugs acting with hub genes.
Abbreviations
IPAH: Idiopathic pulmonary arterial hypertension miRNA: microRNA
DEGs: Differentially expressed genes
DEMIs: Differentially expressed miRNAs
GEO: Gene Expression Omnibus
GO: Gene Ontology
KEGG: Kyoto Encyclopedia of Genes and Genomes
PPI: $\quad$ Protein-protein interaction
BP: Biological processes
CC: $\quad$ Cellular components
MF: Molecular function
PAP: $\quad$ Pulmonary artery pressure
PVR: $\quad$ Pulmonary vascular resistance
PDE5: $\quad$ Phosphodiesterase type 5
PASMC: Pulmonary vascular smooth muscle cell
EDN1: Endothelin-1
VCAM1: Vascular cell adhesion molecule 1
AQP9: Aquaporin 9
SPP1: $\quad$ Secreted phosphoprotein 1
SAA1: Serum amyloid A1.

\section{Data Availability}

All data generated or analysed during this study are included in this published article [and its supplementary information files].

\section{Conflicts of Interest}

The authors declare that they have no competing interests.

\section{Authors' Contributions}

All authors made substantive intellectual contributions to this study to qualify as authors. Ruizheng Shi conceived of the design of the study. Chan Li performed the study, collected the data, and contributed to the design of the study. Chan Li and Zeyu Zhang drafted the manuscript. Qian Xu revised the manuscript. All authors read and approved the final manuscript.

\section{Acknowledgments}

The work was supported by grants from the National Nature Science Foundation of China (No. 81570453 to S.R.Z.) and the Chinese Cardiovascular Association V.G foundation (No. 2017-CCA-VG-005 to S.R.Z).

\section{Supplementary Materials}

Supplement Table 1: the 30 DEGs in the lung tissue of patients with IPAH. Supplement Table 2: the 6 DEMIs in the lung tissue of patients with IPAH. Supplement Figure 1: the top 10 enriched Gene Ontology terms in biological process (A), cellular component (B), molecular function (C), and enriched Kyoto Encyclopedia of Genes and Genomes pathway (D) of the targets of DEMIs. DEMIs: differential expressed miRNAs. (Supplementary Materials)

\section{References}

[1] A. L. Firth, J. Mandel, and J. X. J. Yuan, "Idiopathic pulmonary arterial hypertension," Disease Models \& Mechanisms, vol. 3, no. 5-6, pp. 268-273, 2010.

[2] E. M. T. Lau, E. Giannoulatou, D. S. Celermajer, and M. Humbert, "Epidemiology and treatment of pulmonary arterial hypertension," Nature Reviews Cardiology, vol. 14, no. 10, pp. 603-614, 2017.

[3] H. Matsubara and A. Ogawa, "Treatment of idiopathic/hereditary pulmonary arterial hypertension," Journal of Cardiology, vol. 64, no. 4, pp. 243-249, 2014.

[4] D. B. Taichman and J. Mandel, "Epidemiology of pulmonary arterial hypertension," Clinics in Chest Medicine, vol. 34, no. 4, pp. 619-637, 2013.

[5] N. Nickel, H. Golpon, M. Greer et al., "The prognostic impact of follow-up assessments in patients with idiopathic pulmonary arterial hypertension," The European Respiratory Journal, vol. 39, no. 3, pp. 589-596, 2012.

[6] J. D. Christie, L. B. Edwards, P. Aurora et al., "Registry of the International Society for Heart and Lung Transplantation: twenty-fifth official adult lung and heart/lung transplantation 
report-2008," The Journal of Heart and Lung Transplantation, vol. 27, no. 9, pp. 957-969, 2008.

[7] D. P. Bartel, "MicroRNAs: genomics, biogenesis, mechanism, and function," Cell, vol. 116, no. 2, pp. 281-297, 2004.

[8] J. Bienertova-Vasku, J. Novak, and A. Vasku, "MicroRNAs in pulmonary arterial hypertension: pathogenesis, diagnosis and treatment," Journal of the American Society of Hypertension, vol. 9, no. 3, pp. 221-234, 2015.

[9] C. J. Rhodes, J. Wharton, R. A. Boon et al., "Reduced microRNA-150 is associated with poor survival in pulmonary arterial hypertension," American Journal of Respiratory and Critical Care Medicine, vol. 187, no. 3, pp. 294-302, 2013.

[10] A. Courboulin, R. Paulin, N. J. Giguère et al., "Role for miR204 in human pulmonary arterial hypertension," The Journal of Experimental Medicine, vol. 208, no. 3, pp. 535-548, 2011.

[11] K. Yuan, M. Orcholski, X. Tian, X. Liao, and V. A. de Jesus Perez, "MicroRNAs: promising therapeutic targets for the treatment of pulmonary arterial hypertension," Expert Opinion on Therapeutic Targets, vol. 17, no. 5, pp. 557-564, 2013.

[12] Q. Wang, J. Chen, A. Wang et al., "Differentially expressed circRNAs in melanocytes and melanoma cells and their effect on cell proliferation and invasion," Oncology Reports, vol. 39, no. 4, pp. 1813-1824, 2018.

[13] M. Zeng, J. Liu, W. Yang et al., "Identification of key biomarkers in diabetic nephropathy via bioinformatic analysis," Journal of Cellular Biochemistry, vol. 120, no. 5, pp. 86768688, 2018.

[14] J. L. Deng, Y. H. Xu, and G. Wang, "Identification of potential crucial genes and key pathways in breast cancer using bioinformatic analysis," Frontiers in Genetics, vol. 10, p. 695, 2019.

[15] T. Wang, X. Zheng, R. Li et al., "Integrated bioinformatic analysis reveals YWHAB as a novel diagnostic biomarker for idiopathic pulmonary arterial hypertension," Journal of Cellular Physiology, vol. 234, no. 5, pp. 6449-6462, 2019.

[16] M. E. Ritchie, B. Phipson, D. Wu et al., "limma powers differential expression analyses for RNA-sequencing and microarray studies," Nucleic Acids Research, vol. 43, no. 7, p. e47, 2015.

[17] G. Yu, L. G. Wang, Y. Han, and Q. Y. He, “clusterProfiler: an R package for comparing biological themes among gene clusters," OMICS: A Journal of Integrative Biology, vol. 16, no. 5, pp. 284-287, 2012.

[18] P. Timalsina, K. Charles, and A. M. Mondal, "STRING PPI score to characterize protein subnetwork biomarkers for human diseases and pathways," in 2014 IEEE International Conference on Bioinformatics and Bioengineering, Boca Raton, FL, USA, November 2014.

[19] N. T. Doncheva, J. H. Morris, J. Gorodkin, and L. J. Jensen, "Cytoscape StringApp: network analysis and visualization of proteomics data," Journal of Proteome Research, vol. 18, no. 2, pp. 623-632, 2018.

[20] A. Subramanian, R. Narayan, S. M. Corsello et al., "A next generation connectivity map: L1000 platform and the first 1,000,000 profiles," Cell, vol. 171, no. 6, pp. 1437-1452.e17, 2017.

[21] D. Wu, C. C. Talbot Jr., Q. Liu et al., "Identifying microRNAs targeting Wnt/ $\beta$-catenin pathway in end-stage idiopathic pulmonary arterial hypertension," Journal of Molecular Medicine, vol. 94, no. 8, pp. 875-885, 2016.

[22] W. Tao, W. Sun, H. Zhu, and J. Zhang, "miR-205-5p suppresses pulmonary vascular smooth muscle cell proliferation by targeting MICAL2-mediated Erk1/2 signaling," Microvascular Research, vol. 124, pp. 43-50, 2019.

[23] P. M. George, R. Badiger, D. Shao et al., "Viral toll like receptor activation of pulmonary vascular smooth muscle cells results in endothelin-1 generation; relevance to pathogenesis of pulmonary arterial hypertension," Biochemical and Biophysical Research Communications, vol. 426, no. 4, pp. 486-491, 2012.

[24] M. Yanagisawa, H. Kurihara, S. Kimura et al., "A novel potent vasoconstrictor peptide produced by vascular endothelial cells," Nature, vol. 332, no. 6163, pp. 411-415, 1988.

[25] V. Biasin, K. Chwalek, J. Wilhelm et al., "Endothelin-1 driven proliferation of pulmonary arterial smooth muscle cells is c-fos dependent," The International Journal of Biochemistry \& Cell Biology, vol. 54, pp. 137-148, 2014.

[26] J. L. Wilson, R. Warburton, L. Taylor, D. Toksoz, N. Hill, and P. Polgar, "Unraveling endothelin-1 induced hypercontractility of human pulmonary artery smooth muscle cells from patients with pulmonary arterial hypertension," PLoS One, vol. 13, no. 4, article e0195780, 2018.

[27] J. C. Huetsch, J. Walker, C. Undem et al., "Rho kinase and $\mathrm{Na}(+) / \mathrm{H}(+)$ exchanger mediate endothelin-1-induced pulmonary arterial smooth muscle cell proliferation and migration," Physiological Reports, vol. 6, no. 9, article e13698, 2018.

[28] C. C. Lin, W. N. Lin, W. C. Hou, L. D. Hsiao, and C. M. Yang, "Endothelin-1 induces VCAM-1 expression-mediated inflammation via receptor tyrosine kinases and Elk/p300 in human tracheal smooth muscle cells," American Journal of Physiology. Lung Cellular and Molecular Physiology, vol. 309, no. 3, pp. L211-L225, 2015.

[29] M. Le Hiress, L. Tu, N. Ricard et al., "Proinflammatory signature of the dysfunctional endothelium in pulmonary hypertension. role of the macrophage migration inhibitory factor/CD74 complex," American Journal of Respiratory and Critical Care Medicine, vol. 192, no. 8, pp. 983-997, 2015.

[30] C. Xue, M. Sowden, and B. C. Berk, "Extracellular cyclophilin a, especially acetylated, causes pulmonary hypertension by stimulating endothelial apoptosis, redox stress, and inflammation," Arteriosclerosis, Thrombosis, and Vascular Biology, vol. 37, no. 6, pp. 1138-1146, 2017.

[31] S. Saadoun, M. C. Papadopoulos, M. Hara-Chikuma, and A. S. Verkman, "Impairment of angiogenesis and cell migration by targeted aquaporin-1 gene disruption," Nature, vol. 434, no. 7034, pp. 786-792, 2005.

[32] S. Gräf, M. Haimel, M. Bleda et al., "Identification of rare sequence variation underlying heritable pulmonary arterial hypertension," Nature Communications, vol. 9, no. 1, article 1416, 2018.

[33] C. Schuoler, T. J. Haider, C. Leuenberger et al., “Aquaporin 1 controls the functional phenotype of pulmonary smooth muscle cells in hypoxia-induced pulmonary hypertension," Basic Research in Cardiology, vol. 112, no. 3, p. 30, 2017.

[34] M. Saker, L. Lipskaia, E. Marcos et al., "Osteopontin, a key mediator expressed by senescent pulmonary vascular cells in pulmonary hypertension," Arteriosclerosis, Thrombosis, and Vascular Biology, vol. 36, no. 9, pp. 1879-1890, 2016.

[35] L. Meng, X. Liu, X. Teng et al., “Osteopontin plays important roles in pulmonary arterial hypertension induced by systemic-to-pulmonary shunt," The FASEB Journal, vol. 33, no. 6, pp. 7236-7251, 2019.

[36] T. Li, X. J. Luo, E. L. Wang et al., "Magnesium lithospermate B prevents phenotypic transformation of pulmonary arteries in 
rats with hypoxic pulmonary hypertension through suppression of NADPH oxidase," European Journal of Pharmacology, vol. 847, pp. 32-41, 2019.

[37] M. Mura, M. J. Cecchini, M. Joseph, and J. T. Granton, “Osteopontin lung gene expression is a marker of disease severity in pulmonary arterial hypertension," Respirology, vol. 24, no. 11, pp. 1104-1110, 2019.

[38] V. Foris, G. Kovacs, M. Tscherner, A. Olschewski, and H. Olschewski, "Biomarkers in pulmonary hypertension: what do we know?," Chest, vol. 144, no. 1, pp. 274-283, 2013.

[39] Y. Hoshikawa, Y. Matsuda, M. Sakuma, and T. Kondo, "Potential therapeutic target for pulmonary arterial hypertensionosteopontin," Nihon Rinsho. Japanese Journal of Clinical Medicine, vol. 66, no. 11, pp. 2097-2101, 2008.

[40] Y. Li, Z. J. Zhuo, H. Zhou et al., " $m i R-34 b / c$ rs4938723 T>C decreases neuroblastoma risk: a replication study in the Hunan children," Disease Markers, vol. 2019, Article ID 6514608, 6 pages, 2019.

[41] X. Liu, J. Feng, L. Tang, L. Liao, Q. Xu, and S. Zhu, "The regulation and function of miR-21-FOXO3a-miR-34b/c signaling in breast cancer," International Journal of Molecular Sciences, vol. 16, no. 2, pp. 3148-3162, 2015.

[42] X. Lin, F. Li, F. Xu et al., "Aberration methylation of miR-34b was involved in regulating vascular calcification by targeting Notch1," Aging, vol. 11, no. 10, pp. 3182-3197, 2019.

[43] C. M. Uhlar and A. S. Whitehead, "Serum amyloid A, the major vertebrate acute-phase reactant," European Journal of Biochemistry, vol. 265, no. 2, pp. 501-523, 1999.

[44] B. D. Johnson, K. E. Kip, O. C. Marroquin et al., "Serum amyloid $\mathrm{A}$ as a predictor of coronary artery disease and cardiovascular outcome in women: the National Heart, Lung, and Blood Institute-Sponsored Women's Ischemia Syndrome Evaluation (WISE)," Circulation, vol. 109, no. 6, pp. 726-732, 2004.

[45] X. Xie, Y. T. Ma, Y. N. Yang et al., "Polymorphisms in the SAA1/2 gene are associated with carotid intima media thickness in healthy Han Chinese subjects: the cardiovascular risk survey," PLoS One, vol. 5, no. 11, article e13997, 2010.

[46] Y. Kumon, T. Suehiro, K. Hashimoto, and J. D. Sipe, "Dexamethasone, but not IL-1 alone, upregulates acute-phase serum amyloid a gene expression and production by cultured human aortic smooth muscle cells," Scandinavian Journal of Immunology, vol. 53, no. 1, pp. 7-12, 2001.

[47] S. Rich, E. Kaufmann, and P. S. Levy, "The effect of high doses of calcium-channel blockers on survival in primary pulmonary hypertension," The New England Journal of Medicine, vol. 327, no. 2, pp. 76-81, 1992.

[48] B. I. Medarov and M. A. Judson, "The role of calcium channel blockers for the treatment of pulmonary arterial hypertension: how much do we actually know and how could they be positioned today?" Respiratory Medicine, vol. 109, no. 5, pp. 557-564, 2015. 